\section{Consumo alimentar e antropometria relacionados à síndrome de fragilidade em idosos residentes em comunidade de baixa renda de um grande centro urbano}

\section{Food consumption and anthropometry related to the frailty syndrome in low-income community- living elderly in a large city}

Consumo alimentario y antropometría, relacionados con el síndrome de fragilidad en ancianos, residentes en una comunidad de renta baja de un gran centro urbano

\author{
Amanda de Carvalho Mello 1 \\ Marilia Sá Carvalho 2 \\ Luciana Correia Alves 3 \\ Viviane Pereira Gomes 1 \\ Elyne Montenegro Engstrom 1
}

doi:10.1590/0102-311X00188815

\section{Resumo}

O objetivo deste estudo foi descrever dados antropométricos e de alimentação relacionados à síndrome de fragilidade em idosos. O desenho foi transversal, com indivíduos $\geq 60$ anos de inquérito domiciliar realizado em Manguinhos, Município do Rio de Janeiro, Brasil $(n=137)$. Foram obtidos o diagnóstico de sindrome de fragilidade segundo Fried et al., medidas antropométricas e aplicado questionário de frequência de consumo alimentar, comparando-se às recomendações do Ministério da Saúde. Nos grupos pré-frágeis e frágeis, o indice de massa corporal e medidas de centralização de gordura apresentaram valores mais elevados e os parâmetros musculares, valores menores, com a gradação da sindrome. O consumo de cereais foi maior nos frágeis e o de feijão e frutas menor; o de vegetais, laticínios e alimentos ricos em açúcar e gordura foi maior nos pré-frágeis; o de carne foi semelhante nos grupos. Assim, o diagnóstico da síndrome, a avaliação antropométrica e da alimentação são ações a serem incluídas às políticas de saúde do idoso por identificar precocemente o risco e beneficiar intervenções de prevenção e promoção à saúde e nutrição.

Idoso Fragilizado; Consumo de Alimentos; Antropometria; Avaliação Nutricional

\author{
Correspondência \\ A. C. Mello \\ Trav. Tertuliano Pimenta 157, Nova Iguaçu, RJ 26256-170, \\ Brasil. \\ amandita.nut@gmail.com \\ 1 Escola Nacional de Saúde Pública Sergio Arouca, Fundação \\ Oswaldo Cruz, Rio de Janeiro, Brasil. \\ 2 Programa de Computação Científica, Fundação Oswaldo \\ Cruz, Rio de Janeiro, Brasil. \\ 3 Universidade Estadual de Campinas, Campinas, Brasil.
}




\section{Introdução}

Nas últimas décadas, as mudanças demográficas brasileiras vêm determinando alterações na estrutura etária da população, caracterizada por um aumento progressivo e acentuado da população adulta e, especialmente, a idosa. A queda dos níveis de fecundidade combinada à redução da mortalidade têm acarretado um processo de envelhecimento populacional e de aumento da longevidade da população no Brasil 1. Interligado à transição demográfica, o país tem experimentado um processo de transição epidemiológica, marcado pelo aumento da participação das doenças crônico-degenerativas em substituição às doenças infectocontagiosas no total de mortes ${ }^{2}$. Assim, a tendência atual é ter um número crescente de indivíduos que vivem mais e apresentam maior número de condições crônicas.

A síndrome de fragilidade caracteriza-se pela redução da reserva energética e da resistência fisiológica, com um declínio cumulativo de órgãos e sistemas corporais e consequentes alterações neuromusculares, desregulação do sistema endócrino e disfunção do sistema imunológico 3,4. No Brasil, a prevalência varia de $10 \%$ a $25 \%$, enquanto em chineses, americanos e europeus é de 4,9\%, 6,9\% e $17 \%$, respectivamente 5,6. É, por ora, considerada um dos grandes problemas enfrentados pelas sociedades que envelhecem, por afetar a qualidade de vida dos idosos e estar diretamente associada a desfechos negativos em saúde, como as quedas, fraturas, hospitalização e institucionalização 7,8.

Em relação às principais doenças crônicas, como as cardiovasculares, câncer, diabetes, enfermidades respiratórias e neuropsiquiátricas, estudos têm mostrado uma forte associação com o consumo de tabaco e álcool, excesso de peso, baixo consumo de frutas e verduras e sedentarismo, sendo o monitoramento da prevalência das doenças e seus fatores de risco basilar para definição de políticas de saúde voltadas à prevenção desses agravos ${ }^{9}$. No entanto, a relação de características ligadas à alimentação com a síndrome de fragilidade em idosos ainda é pouco estudada, como evidenciado em revisão sistemática acerca de fatores associados à síndrome 10 .

A perda de massa muscular interagindo com a nutrição deficiente caracterizam um ciclo autossustentado, com potencial decrescente de energia além do observado como normal do envelhecimento, levando à redução da atividade física e consequente subnutrição crônica, sarcopenia, redução da força física e do gasto total de energia 11,12.

A perda de massa muscular pode ser identificada por meio de uma abordagem mais abrangente, factível de execução nos serviços de saúde, que inclui avaliação do consumo alimentar e medidas antropométricas que mensuram não somente o peso total, mas buscam diferenciar a massa muscular da gordura corporal. Medidas simples de adiposidade central como circunferência da cintura, relação cintura-quadril e índice de conicidade, e indicadores antropométricos de massa muscular como circunferência muscular do braço e da panturrilha, poderiam ser avaliadas na rotina de atendimento para se ter mais precisão do quadro nutricional do idoso. A investigação mais detalhada dos hábitos alimentares, levando em consideração a quantidade, variedade, disponibilidade de alimentos, condições, procedimentos e preparo também possibilitaria uma intervenção dietoterápica mais eficiente 13,14 . Isso permitiria maior potência na prevenção de doenças relacionadas à alimentação e ao acúmulo excessivo de gordura abdominal, tais como as crônico-degenerativas, e aquelas relacionadas à diminuição dos estoques de proteína, como a incapacidade funcional e síndrome de fragilidade 4,15 .

Sendo assim, o objetivo deste estudo foi estudar a síndrome de fragilidade em idosos moradores de uma comunidade de contexto socioeconômico e ambiental vulnerável e a relação dela com parâmetros antropométricos e de consumo alimentar.

\section{Materiais e métodos}

\section{Desenho do estudo e amostra}

Este é um estudo transversal, de base domiciliar, cujos participantes (idosos de 60 anos ou mais de idade, ambos os sexos) foram identificados pelo Inquérito sobre Condições de Saúde e Utilização de Serviços de Saúde no Território de Manguinhos, Município do Rio de Janeiro, Brasil, estudo mais abrangente em uma área de favelas realizado no período de 2012-2013 16. 
O referido inquérito teve amostra probabilística de domicílios $(\mathrm{n}=955)$ e moradores $(\mathrm{n}=2.918)$, com seleção sistemática de um domicílio a cada dez, de modo que toda a comunidade tivesse igual probabilidade de ser entrevistada. Todos os idosos identificados no inquérito foram convidados a participar de etapa complementar da pesquisa, com coleta de informações adicionais do presente estudo. Houve a aplicação de questionários domiciliar e individual compostos por questões fechadas, construído por perguntas extraídas da Pesquisa Nacional de Saúde (PNS) 17 e da Pesquisa Nacional por Amostra de Domicílios (PNAD) 18.

O tamanho amostral foi validado pela equação de Lwanga \& Lemeshow 19 , sendo $\mathrm{N}=\mathrm{P} \mathrm{x}$ $\mathrm{Q} / \mathrm{E} / 1,96)^{2}$, onde: $\mathrm{N}$ = tamanho mínimo da amostra; $\mathrm{P}=$ prevalência média de síndrome de fragilidade em idosos maiores de 65 anos, segundo o Ministério da Saúde em 2006 5; Q = 100 - P; E = margem de erro amostral tolerado. Considerou-se um erro amostral de $\pm 7 \%$ e confiabilidade do estudo em $95 \%$. Assim: $\mathrm{N}=[17,5 \mathrm{x}(100-17,5)] /(7 / 1,96) 2=1443,75 / 12,75=113$ sujeitos. Adicionando-se $5 \%$ de perda, deveriam ser selecionados 119 idosos.

A seleção dos participantes teve como critério de inclusão idosos de ambos os sexos, com 60 anos ou mais de idade e residentes nos domicílios sorteados pelo Inquérito de Saúde-Manguinhos. Foram excluídos do estudo aqueles que se encontravam acamados ou cadeirantes, pois não poderiam realizar um dos testes (velocidade da marcha) que faz parte do critério diagnóstico adotado para a síndrome. As entrevistas e avaliações ocorreram nas unidades básicas e/ou nos domicílios dos participantes por dois pesquisadores treinados e equipamentos calibrados.

\section{Variáveis estudadas}

A síndrome de fragilidade foi avaliada segundo o critério proposto por Fried et al. 4 , composto por cinco itens: perda de peso não intencional de pelo menos $4,5 \mathrm{~kg}$ ou pelo menos $5 \%$ do peso corporal no último ano, fadiga autorreferida, força diminuída de preensão palmar medida com um dinamômetro na mão dominante, baixo nível de atividade física medido pelo dispêndio semanal de energia em Kcal, redução da velocidade da marcha em segundos.

A perda de peso foi analisada de acordo com o relato do entrevistado. A fadiga foi considerada mediante resposta afirmativa em pelo menos uma de duas perguntas da escala de depressão do Center for Epidemiological Studies (CES-D) 20, versão validada em idosos brasileiros 21. A força diminuída de preensão foi estimada com dinamômetro do tipo JAMAR, modelo NC 701/42 (North Coast Medical Inc., Gilroy, Estados Unidos), e foram considerados como apresentando síndrome de fragilidade para esse critério os idosos que tiveram medidas abaixo do percentil 20 de força de preensão dentro de cada quartil de índice de massa corporal (IMC) da amostra, ajustado por sexo. A atividade física foi investigada por meio da versão reduzida do Questionário Internacional de Atividade Física (International Physical Activity Questionnaire - IPAQ) e foram definidos como apresentando baixos níveis aqueles que tiveram dispêndio semanal abaixo do percentil 20 de calorias da amostra, ajustado por sexo. A velocidade da marcha foi mensurada pela cronometragem do tempo de marcha em segundos gasto para percorrer uma distância de 4,6 metros em velocidade habitual, e foi considerada velocidade baixa aqueles que revelaram tempo acima do percentil 80 ajustado por sexo e média de estatura.

Os idosos apresentando pelo menos três dos cinco itens foram considerados frágeis. Aqueles com presença de um a dois componentes foram classificados como pré-frágeis e os que não preencheram nenhum dos critérios foram considerados não frágeis.

Para avaliação antropométrica, foram aferidas massa corporal (MC), estatura, circunferência da cintura (CC), do braço (CB), da panturrilha (CP), dobra cutânea triciptal (DCT), e calculados o IMC, a circunferência muscular do braço (CMB), a relação cintura-estatura (RCE), e o índice de conicidade (IC). Todas as medidas foram aferidas segundo protocolo da Organização Mundial da Saúde (OMS) 22 e de acordo com as técnicas preconizadas por Gomes et al. 23 e Harrison et al. 24. O IMC foi calculado pela fórmula: $\mathrm{IMC}=\mathrm{MC}(\mathrm{kg}) /$ estatura $2\left(\mathrm{~m}^{2}\right)$. A CMB foi obtida pela equação: $\mathrm{CMB}(\mathrm{cm})=[\mathrm{CB}(\mathrm{cm})-(\pi \mathrm{x}$ $\operatorname{DCT}(\mathrm{cm}))] 25$. A RCE foi determinada pela fórmula: $R C E=C C(\mathrm{~cm}) /$ estatura $(\mathrm{cm})$, e o IC pela expressão: $\mathrm{IC}=\mathrm{CC}(\mathrm{m}) /(0,109) 26,27$.

A avaliação do consumo alimentar foi feita por meio da aplicação de um Questionário de Frequência de Consumo de Alimentos (QFCA), de natureza quantitativa e qualitativa, que computou a ingestão diária, semanal e mensal de uma lista de alimentos. Esse QFCA foi baseado na proposta de Sichieri 28, com 83 
itens, validado para adultos da cidade do Rio de Janeiro. Os dados obtidos foram distribuídos em grupos alimentares e convertidos em unidades e frações de porções de consumo diárias. As porções estimadas foram comparadas com as porções sugeridas pelo Ministério da Saúde, no guia Alimentação Saudável para a Pessoa Idosa - Um Manual para Profissionais de Saúde 29.

\section{Análise estatística}

A análise da síndrome de fragilidade com as variáveis sociodemográficas e parâmetros antropométricos e alimentares foi feita por meio de proporções para as variáveis categóricas (Tabela 1) e média e intervalo de mais ou menos um erro-padrão para as variáveis contínuas (Figuras 1 e 2).

Os dados foram armazenados no software EpiData (Epidata Association, Odense, Dinamarca) e as análises empreendidas no pacote estatístico R versão 3.0.2 (The R Foundation for Statistical Computing, Viena, Áustria; http://www.r-project.org).

\section{Aspectos éticos}

Este estudo foi aprovado pelo Comitê de Ética em Pesquisa da Escola Nacional de Saúde Pública Sergio Arouca, Fundação Oswaldo Cruz, parecer no 7933/2012, e todos os participantes assinaram o Termo de Consentimento Livre e Esclarecido (TCLE).

\section{Tabela 1}

Frequência das características sociodemográficas segundo a classificação de síndrome de fragilidade em idosos de Manguinhos, Rio de Janeiro, Brasil, 2012-2013.

\begin{tabular}{|c|c|c|c|c|}
\hline \multirow[t]{2}{*}{ Variáveis } & \multicolumn{4}{|c|}{ Fragilidade } \\
\hline & $\begin{array}{c}\text { Não frágil } \\
\text { n (\%) }\end{array}$ & $\begin{array}{c}\text { Pré-frágil } \\
\text { n (\%) }\end{array}$ & $\begin{array}{l}\text { Frágil } \\
\text { n (\%) }\end{array}$ & $\begin{array}{l}\text { Total } \\
\text { n (\%) }\end{array}$ \\
\hline \multicolumn{5}{|l|}{ Idade (anos) * } \\
\hline $60-69$ & $25(69,4)$ & $44(52,4)$ & $6(35,3)$ & $75(54,8)$ \\
\hline $70-79$ & $9(25,0)$ & $30(35,7)$ & $5(29,4)$ & $44(32,1)$ \\
\hline 80 e mais & $2(5,6)$ & $10(11,9)$ & $6(35,3)$ & $18(13,1)$ \\
\hline \multicolumn{5}{|l|}{ Sexo } \\
\hline Feminino & $22(61,1)$ & $57(67,9)$ & $14(82,4)$ & $93(67,9)$ \\
\hline Masculino & $14(38,9)$ & $27(32,1)$ & $3(17,6)$ & $44(32,1)$ \\
\hline \multicolumn{5}{|l|}{ Escolaridade (anos de estudo) } \\
\hline Menos de 1 & $5(13,9)$ & $16(19,05)$ & $6(35,3)$ & $27(19,7)$ \\
\hline $1-3$ & $9(25,0)$ & $28(33,3)$ & $3(17,6)$ & $40(29,2)$ \\
\hline $4-7$ & $10(27,8)$ & $23(27,4)$ & $7(41,2)$ & $40(29,2)$ \\
\hline 8 ou mais & $11(30,5)$ & $16(19,05)$ & $1(5,9)$ & $28(20,4)$ \\
\hline Não soube responder & $1(2,8)$ & $1(1,2)$ & $0(0,0)$ & $2(1,5)$ \\
\hline \multicolumn{5}{|l|}{ Renda mensal per capita ** } \\
\hline Até um salário mínimo & $15(60,0)$ & $50(73,5)$ & $14(87,5)$ & $79(72,5)$ \\
\hline Mais de um salário mínimo & $10(40,0)$ & $18(26,5)$ & $2(12,5)$ & $30(27,5)$ \\
\hline Total & $36(26,3)$ & $84(61,3)$ & $17(12,4)$ & $137(100,0)$ \\
\hline
\end{tabular}

* Teste qui-quadrado - $\mathrm{p}<0,05$;

** Dados faltantes por ausência de resposta do entrevistado.

Fonte: elaboração própria. 


\section{Figura 1}

Médias de parâmetros antropométricos segundo a classificação de síndrome de fragilidade dos idosos de Manguinhos, Rio de Janeiro, Brasil, 2013.

1a) Índice de massa corporal

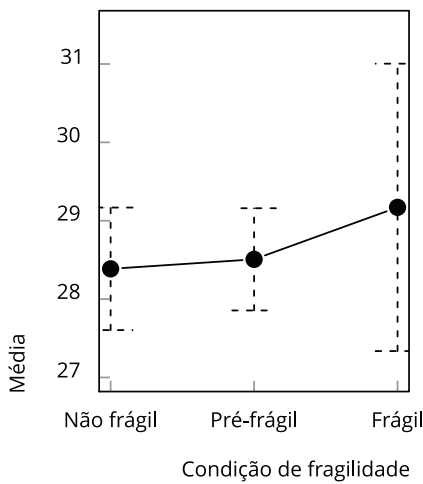

1d) Circunferência da cintura

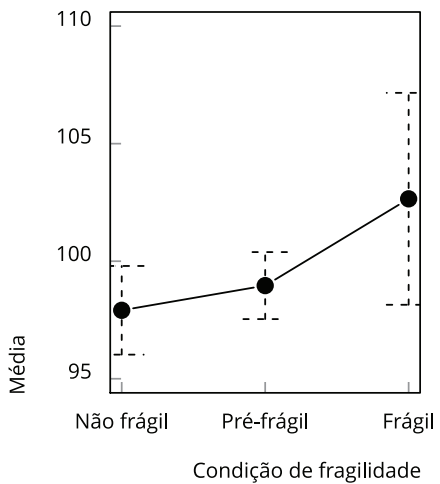

1b) Circunferência muscular de braço

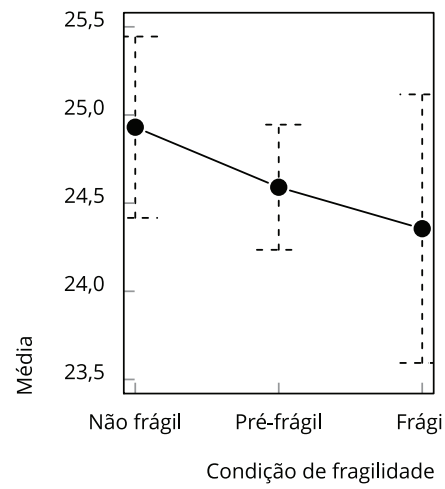

1e) Relação cintura-estatura

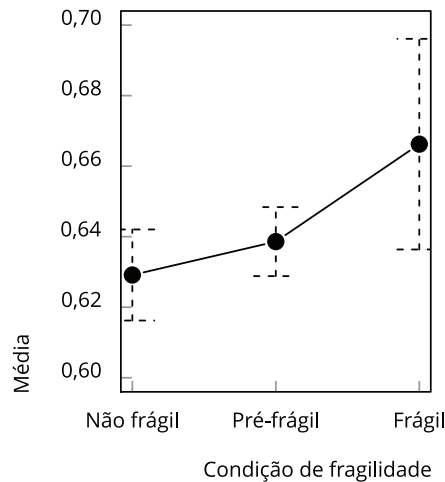

1c) Circunferência de panturrilha

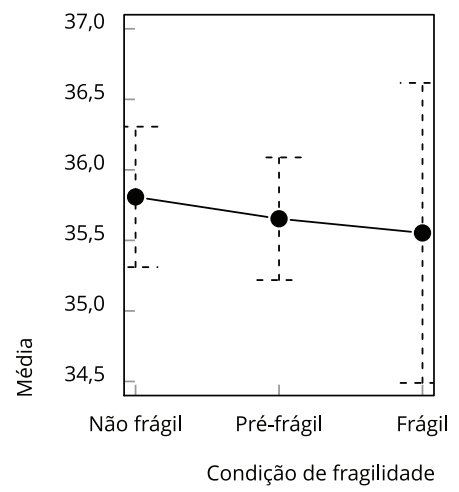

1f) Índice de conicidade

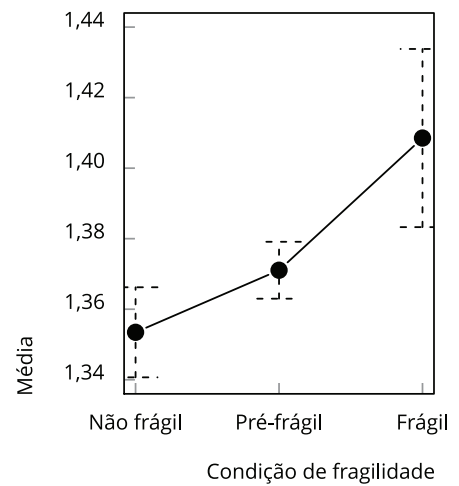

\section{Resultados}

Foram estudados 137 idosos, sendo 44 homens (32,1\%) e 93 mulheres (67,9\%). A idade variou de 61-97 anos, com mediana de 69,0 anos e média e desvio-padrão de 70,2 e 7,4 anos, respectivamente. Dos idosos convidados a integrar o estudo, $2 \%$ foram excluídos de acordo com os critérios de elegibilidade e $6,5 \%$ se recusaram a participar. A maioria relatou não estar trabalhando $(77,4 \%)$, ter de um a sete anos de estudo (60\%) e receber até um salário mínimo corrente da época $(\mathrm{R} \$ 622,00)(72,5 \%)$, o que evidencia um perfil de população de baixa renda e escolaridade. A Tabela 1 apresenta as frequências das principais características sociodemográficas segundo a síndrome de fragilidade.

Quanto à condição de síndrome de fragilidade, 12,4\% dos idosos foram considerados frágeis e 61,3\% pré-frágeis. A única variável significativamente associada à classificação da síndrome foi a idade. No que se refere à frequência dos componentes do fenótipo da síndrome, destacam-se: perda de peso em 20,4\% dos idosos ( $n=28)$; fadiga em 33,6\% ( $n=46)$; e baixo nível de atividade física, força diminuída de preensão palmar e velocidade de marcha em, respectivamente, $20,4 \%, 23,4 \%$ e $21,2 \%$.

Na Figura 1, observa-se que, embora os valores de IMC sejam maiores com a gradação da síndrome, os parâmetros de aferição de massa muscular ( $\mathrm{CMB}$ e $\mathrm{CP}$ ) tendem a ser menores e os de medidas 
Figura 2

Médias de consumo de porções diárias de grupos alimentares segundo a classificação de síndrome de fragilidade dos idosos de Manguinhos, Rio de Janeiro, Brasil, 2013.
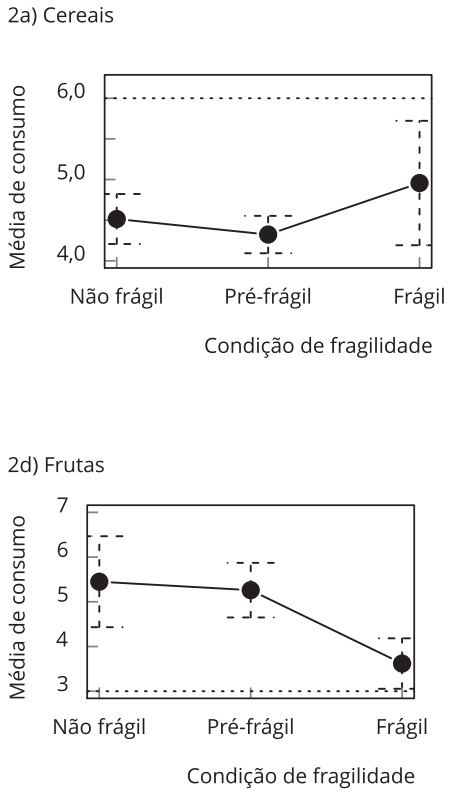

2g) Ricos em açúcares e gorduras

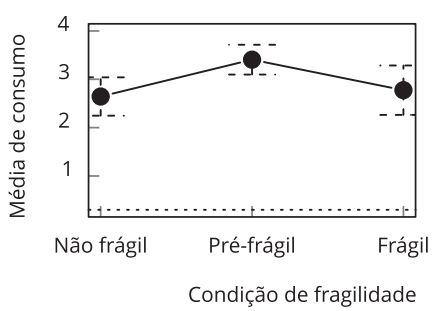

2b) Feijões
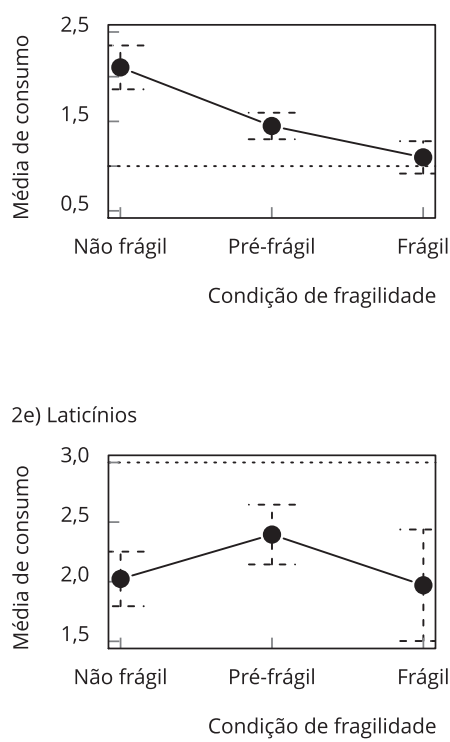

2c) Verduras e legumes
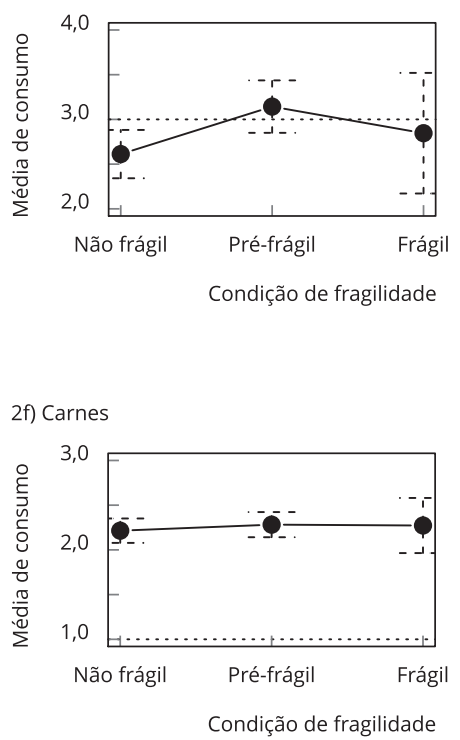

Nota: linha horizontal tracejada: recomendação do Ministério da Saúde 29.

de tendência central (CC, RCE e IC), a ser mais elevados nos grupos pré-frágeis e frágeis, mesmo com a quantidade pequena de idosos frágeis na amostra, evidenciada pela ampliação do intervalo em torno da média do parâmetro.

A Figura 2 apresenta a média de consumo de porções diárias dos grupos alimentares segundo a classificação da síndrome. A linha horizontal tracejada representa a recomendação do Ministério da Saúde 29 para cada grupo e a linha vertical tracejada em torno do ponto, o intervalo referente ao erropadrão. Em relação às recomendações, verifica-se que todos os idosos superaram o consumo recomendado para frutas, carnes e, sobretudo, alimentos ricos em açúcares e gorduras, e não alcançaram o sugerido para laticínios e cereais. Em relação às verduras e legumes, verifica-se que os não frágeis não alcançaram a recomendação proposta. Quanto aos feijões, todos atingiram a recomendação. Comparando-se os grupos, o consumo de cereais tende a ser maior no grupo frágil; o de feijões tende a ser menor quão mais grave é a síndrome, assim como o de frutas; o consumo de verduras e legumes, laticínios e alimentos ricos em açúcares e gorduras têm seu pico de consumo no grupo dos pré-frágeis; e o consumo de carne tende a ser semelhante nos três grupos. 


\section{Discussão}

O presente estudo encontrou uma elevada prevalência das condições de pré-fragilidade e fragilidade, sendo superior em mulheres e na idade mais avançada, uma tendência de IMC mais elevado nos grupos pré-frágeis e frágeis e de menor consumo de feijões e frutas com a gradação da síndrome nessa amostra. Cabe ressaltar que a pesquisa ocorreu em área favelizada, com um dos piores Índices de Desenvolvimento Humano do Estado do Rio de Janeiro, o que retrata uma condição de maior vulnerabilidade dos residentes. Além de o idoso ter dificuldades inerentes ao próprio processo de envelhecimento, as pessoas que residem em favelas vivem num contexto social histórico de urbanização e saneamento precários, falta de investimentos em educação, habitação e saúde, desigualdade social, baixa qualidade de vida e violência, com escassez de pesquisas, particularmente as relacionadas ao consumo alimentar e síndromes associadas ao avanço da idade.

Tal prevalência difere da grande parte dos estudos internacionais que utilizaram o mesmo método diagnóstico da síndrome, em que os índices variam de 34,6\% a 50,4\% para pré-frágeis e de 4,9\% a 17\% para frágeis 6 . Contudo, em áreas de condições socioeconômicas piores, a prevalência se assemelha, como no caso de estudo realizado no Nordeste do Brasil, em que as prevalências foram de 60,1\% para pré-frágeis e $17,1 \%$ para frágeis 30 . Observa-se também que a frequência de pré-fragilidade é mais elevada em locais com processo de transição demográfica mais recente, como é o caso de Manguinhos e dos países em desenvolvimento quando comparados aos desenvolvidos, dados que ainda não possuem uma quantidade elevada tanto em números absolutos quanto relativos de idosos muitos idosos 31,32. Esse grupo é o mais acometido pela síndrome, que tende a aumentar com o envelhecimento e a maior longevidade da população 33,34.

No que diz respeito à relação entre síndrome de fragilidade e condições nutricionais em idosos não frágeis, pré-frágeis e frágeis, evidenciou-se uma situação adversa nos idosos estudados: nos grupos pré-frageis e frágeis, os valores de IMC e de medidas de centralização de gordura abdominal (indicadores de obesidade) foram mais elevados; os parâmetros de massa muscular apresentaram valores menores (perda de massa magra); assim como o consumo de feijão e de frutas. Há escassez de estudos acerca dessas relações em nosso meio, aspecto inovador e relevante do presente estudo.

O padrão de elevação do IMC nos grupos pré-frágeis e frágeis aqui evidenciado é corroborado em estudos internacionais, nos quais a proporção de mulheres americanas e italianas pré-frágeis e frágeis aumentou com o incremento no IMC 35,36. A utilização de diferentes indicadores antropométricos foi importante e mostrou que a avaliação do estado nutricional somente pelo uso do IMC é insuficiente para um diagnóstico adequado, visto que não detecta perda de massa muscular e centralização de gordura corporal, agravos passíveis de detecção e intervenção precoce. Nos serviços de atenção primária à saúde no Brasil, o Sistema de Vigilância Alimentar e Nutricional (SISVAN) orienta apenas aferição de MC e estatura para cálculo do IMC ${ }^{37}$. Especialmente em idosos, cabe atentar para o uso crítico do IMC para avaliação nutricional devido à tendência de centralização de gordura com o aumento da idade 38 . Além disso, a perda progressiva de massa magra e a regionalização de gordura, presentes no envelhecimento, podem não levar à redução do IMC, o que ocasionaria um diagnóstico equivocado de eutrofia, mascarando, desse modo, um déficit nutricional importante e maior risco de desenvolvimento da síndrome de fragilidade.

Reforçando tal discussão, há estudos que relataram ser frequente em idosos frágeis a presença da chamada obesidade sarcopênica, caracterizada pelo aumento de MC por conta dos depósitos de gordura, concomitantemente à supressão de massa muscular nas extremidades, tornando o idoso mais fragilizado e suscetível a problemas nos membros inferiores, redução de atividade física e quedas 35,39. Dessa forma, adaptações quanto ao uso do IMC e a adoção de outros métodos de avaliação do estado nutricional em idosos são sugeridas há bastante tempo 40 .

Nesse sentido, foram testados outros parâmetros relacionados à mensuração da massa muscular, no caso a CP e CMB, e à centralização de gordura corporal, como a CC, a RCE e o IC, segundo a classificação de síndrome de fragilidade. Percebeu-se que todos os indicadores de massa muscular foram menores com a gradação da síndrome, ocorrendo o contrário com os indicadores de adiposidade abdominal. Esses resultados concordam com os encontrados em outros estudos. Em idosos italianos de ambos os sexos com idade acima de 80 anos, foi constatada uma relação inversa entre medida de $\mathrm{CP}$ e classificação de síndrome de fragilidade ${ }^{41}$. Já em idosos ingleses com 65 anos ou mais de 
idade, verificou-se que aqueles que apresentavam medidas mais elevadas de CC eram mais frágeis 42 . Vale ressaltar que são escassos os estudos associando tais indicadores com a síndrome, especialmente com utilização da RCE e do IC, além do fato de todos os indicadores, exceto o IMC, não ter um ponto de corte específico para idosos, o dificulta um diagnóstico baseado em tais parâmetros, suscitando a realização de pesquisas com esse fim. Os perímetros são medidas fáceis de obter porque necessitam apenas de uma fita métrica, e se utilizados como medidas complementares às de rotina clínica, como peso e estatura, tornam a avaliação de saúde mais informativa para tomada de decisões e prescrições.

Quanto à avaliação do consumo alimentar, ela foi feita mediante o uso do QFCA, convertendose os resultados em quantidade de porções diárias de grupos alimentares. A opção por essa análise objetivou ampliar o debate sobre formas de se gerar informações com base no consumo alimentar que sejam mais práticos para formulação de políticas voltadas para a alimentação saudável e utilização nos serviços de saúde. A sugestão de porções diárias e as instruções simples para alimentação e nutrição têm sido a diretriz adotada pelo Ministério da Saúde para orientação nutricional por meio de guia alimentar específico para tal grupo etário 29 . Essa ação facilita o entendimento e a comunicação entre profissionais e usuários na atenção primária em saúde do que é entendido como alimentação adequada, tornando a informação mais objetiva e promovendo maior adesão às intervenções.

Os estudos que relacionam alimentação com síndrome de fragilidade na literatura científica em geral analisam a associação entre macro e micronutrientes com a síndrome, o que dificulta a comparação com os nossos resultados, ficando, dessa maneira, restrita à análise do comportamento de consumo entre os grupos e sua relação com o agravo.

Ao se compararem os resultados encontrados com as porções diárias sugeridas pelo Ministério da Saúde ${ }^{29}$, observou-se que, para a maioria dos grupos alimentares, os idosos da amostra superaram a recomendação, salvo verduras e legumes, laticínios e cereais. Contudo, a amostra do estudo é de uma região específica (um complexo de favelas), diferente da maioria das pesquisas com essa faixa etária, o que pode gerar padrões de consumo alimentar diferentes do habitualmente encontrado em outras literaturas. Além disso, tais resultados devem ser analisados com cautela quanto à comparação entre o que é recomendado para populações sadias e para idosos em condições de síndrome de fragilidade. Um panorama sobre hábitos de consumo alimentar na população brasileira foi estudado pela PNS, que investigou como marcadores de padrão saudável de alimentação o consumo recomendado de frutas, legumes e verduras e de feijão ${ }^{17}$. No Brasil, o percentual de pessoas de 18 anos ou mais de idade que consumiam cinco porções diárias de frutas e hortaliças foi de 37,3\%, proporção que aumentava com a idade (40\% nos idosos) e com o grau de escolaridade. Já o consumo regular (em cinco ou mais dias da semana) de feijão foi referido por 71,9\% das pessoas (e 71\% dos idosos).

Em relação ao maior consumo de cereais nos grupos frágeis, ressalta-se que esses idosos, em geral, são mais velhos; assim, uma hipótese é a de que com o avanço da idade, há modificações fisiológicas importantes que afetam o consumo alimentar, como a redução das papilas gustativas, diminuição do olfato e da visão e maior degeneração cognitiva, o que diminui o prazer durante as refeições e aumenta a dificuldade em cortar e consumir alimentos mais fibrosos e consistentes, como frutas, verduras e carnes ${ }^{43}$. Isso pode interferir na ingestão e escolha de alimentos, ocasionando a preferência por alimentos menos densos, como raízes cozidas, arroz e pães, presentes no grupo de cereais.

A ingestão reduzida de laticínios chama a atenção pelo fato de esse grupo ser composto de alimentos ricos em cálcio, nutriente que é fundamental para o fortalecimento ósseo e muscular, o que pode influenciar diretamente na síndrome 44.

Observou-se especialmente um consumo elevado de alimentos ricos em açúcares e gorduras saturadas em todos os grupos de participantes, como doces e frituras, contribuindo para a ocorrência de obesidade e doenças crônico-degenerativas. O consumo de carnes também foi superior ao recomendado nos três grupos, especialmente a bovina e a de frango. Embora carnes de uma forma geral sejam boas fontes de proteína de alto valor biológico, deve-se ficar atento se tal consumo provém de carnes magras, pois aquelas ricas em gorduras saturadas também podem ocasionar o aumento de peso e ocorrência de dislipidemias. No presente estudo, igualmente foi verificado um alto relato de consumo de frutas, principalmente laranja, banana e suco de frutas naturais ou de polpa.

Quanto à tendência observada de maior consumo de feijões nos grupos não frágeis e menor consumo com a gradação da síndrome, destaca-se que as leguminosas são alimentos fontes de proteína, o que auxilia na manutenção da massa muscular, de custo mais baixo quando comparado a outras 
fontes proteicas, pobres em gordura e comuns na alimentação brasileira diária 45 . Além disso, têm textura macia e palatabilidade que facilitam seu consumo, dado que os idosos têm maior dificuldade de mastigar em razão da redução da secreção salivar e gástrica e falha na mastigação pela ausência de dentes ou uso de próteses impróprias. Salienta-se que o consumo de arroz com feijão, hábito no Brasil, é considerado mundialmente uma combinação adequada e nutritiva, pois juntos conseguem fornecer a maioria dos aminoácidos essenciais, que são grandes responsáveis na formação dos tecidos musculares e manutenção de diversos sistemas fisiológicos 44 .

A presente pesquisa apresenta como principal contribuição a ampliação da discussão em torno da avaliação nutricional de idosos e sua relação com a síndrome de fragilidade, contribuindo para a reorientação de modelos de atenção que fortaleçam a atenção primária à saúde integral, robusta e resolutiva. Idosos sob risco ou com quadro de síndrome de fragilidade já instalados são grupos prioritários para identificação e manejo resolutivo na atenção primária à saúde, evitando-se consequências indesejáveis, como quedas, fraturas, depressão e dependência funcional, que prejudicam a saúde e o envelhecimento ativo, tanto quanto acarretam ônus cada vez maiores para o sistema de saúde. Os não frágeis e pré-frágeis são ainda sensíveis à prevenção e tratamento que impediriam a progressão para a síndrome, como melhoria da alimentação, uso de suplementos nutricionais e realização de musculação para desacelerar a perda de massa muscular. Tais práticas podem ser fomentadas com o trabalho de equipe multiprofissional, o suporte do Núcleo de Apoio à Saúde da Família, o estímulo ao estilo de vida ativo, a articulação com as Academias da Saúde atuando próximas às unidades básicas, considerando as vulnerabilidades e singularidades dos idosos ${ }^{46}$. No caso daqueles com a síndrome já instalada, essas ações preservariam por mais tempo a qualidade de vida e autonomia do indivíduo.

$\mathrm{Na}$ atenção à saúde dos idosos, é preciso facilitar condições de trabalho e processos de educação em saúde que permitam o diagnóstico precoce de condições comuns na velhice, como desnutrição, sobrepeso, doenças crônico-degenerativas, incapacidade funcional e síndrome de fragilidade. Estudos com esse foco auxiliam na aplicação e validação de métodos simples de avaliação nutricional que podem ser utilizados nos sistemas locais de saúde, na detecção precoce e manejo da síndrome e de agravos nutricionais. Essa avaliação alimentar e nutricional, se incorporada às práticas de saúde no acompanhamento longitudinal da população, especialmente no âmbito da atenção primária à saúde, é potente para promoção de saúde, prevenção e manejo de situações como a síndrome de fragilidade em idosos, mormente em seus estágios iniciais, quando são maiores as chances de regressão do fenômeno 47,48,49. Considerada a porta preferencial de entrada dos usuários na Rede de Atenção à Saúde, a atenção primária à saúde deve prover acesso e resolutividade às necessidades de saúde mais frequentes da população, atuando ainda segundo atributos da integralidade, da longitudinalidade, da coordenação do cuidado das pessoas, da família e da comunidade 46,50 .

Como limitações, cumpre mencionar que o tamanho da amostra é pequeno, ainda que represente em torno de $10 \%$ da população desta faixa etária na região. É importante também ter em vista que o instrumento de avaliação do consumo alimentar utilizado, apesar de validado para a população adulta da cidade do Rio de Janeiro, não incluiu especificamente a população idosa e como instrumento de natureza quanti-qualitativo detalhada, depende da memória do entrevistado sobre seus hábitos alimentares em um tempo determinado. Portanto, seus resultados poderiam subestimar ou superestimar o consumo de determinados alimentos 13 . Somados a isso, avaliação e pontos de corte de alguns componentes da síndrome, como força de preensão, atividade física e velocidade da marcha, sofreram adaptações com o objetivo de se adequar à realidade local da pesquisa, o que pode propiciar algumas divergências ao se comparar com os resultados de outros estudos.

Considerando o desenho transversal do estudo, não é possível estabelecer uma relação de causa e efeito. De fato, o menor consumo de feijão nos grupos frágeis, por exemplo, pode ser posterior à instalação da síndrome, possivelmente por limitações da atividade diária, inclusive cozinhar, caracterizando o efeito de causalidade reversa. Além disso, a própria síndrome é definida como um ciclo autossustentado, em que a redução de força física, modificações alimentares e subnutrição interagem de forma circular. É necessário realizar estudos longitudinais para elucidar melhor a relação entre nutrição e a síndrome de fragilidade, com amostras maiores.

Finalmente, uma alimentação adequada, colorida, equilibrada em nutrientes e rica em fontes de proteína é fundamental para garantir uma boa nutrição e evitar a perda de nutrientes e do tecido muscular, que são fatores relacionados à síndrome de fragilidade. O diagnóstico clínico dos 
componentes que compõem a síndrome e a avaliação antropométrica e do consumo alimentar durante os atendimentos rotineiros nos serviços de saúde se fazem necessários para avaliar com maior precisão a população idosa, garantir uma intervenção precoce e se evitar prejuízos nutricionais e de saúde.

\section{Colaboradores}

A. C. Mello, M. S. Carvalho, L. C. Alves, V. P. Gomes e E. M. Engstrom contribuíram na concepção e projeto, análise e interpretação dos dados, redação e revisão crítica do artigo e aprovação final da versão a ser publicada.

\section{Agradecimentos}

Agradecemos à equipe da Estratégia Saúde da Família de Manguinhos pelo auxílio durante a execução do projeto; à Rede PDTSP-Teias pelo apoio e cessão de dados da pesquisa; às profissionais Ana Paula Josefa da Silva, Cintia da Silva Ramos e Marisa Maria dos Santos na execução da pesquisa de campo; aos voluntários participantes pela contribuição na coleta de dados e à Coordenação de Aperfeiçoamento de Pessoal de Nível Superior (Capes) pela concessão da bolsa de doutorado à A. C. Mello.

\section{Referências}

1. Alves LC, Duarte YAO, Lebrão ML. Perfis de fragilidade dos idosos paulistanos no ano de 2006: evidências do estudo SABE. In: Anais do XIX Encontro Nacional de Estudos Populacionais, ABEP - "População, Governança e Bem-Estar". São Pedro: Associação Brasileira de Estudos Populacionais; 2014. p. 1-19.

2. Instituto Brasileiro de Geografia e Estatística. Mudança demográfica no Brasil no início do século XXI: subsídios para as projeções da população. Rio de Janeiro: Instituto Brasileiro de Geografia e Estatística; 2015.

3. Morley JE, Vellas B, van Kan GA, Anker SD, Bauer JM, Bernabei R, et al. Frailty consensus: a call to action. J Am Med Dir Assoc 2013; 14:392-7.

4. Fried LP, Tangen CM, Walston J, Newman AB, Hirsch C, Gottdiener J, et al. Frailty in older adults: evidence for a phenotype. J Gerontol A Biol Sci Med Sci 2001; 56:M146-56.

5. Ministério da Saúde. Envelhecimento e saúde da pessoa idosa. Brasília: Ministério da Saúde; 2006. (Cadernos de Atenção Básica, 19). (Série A. Normas e Manuais Técnicos).

6. Choi J, Ahn A, Kim S, Won CW. Global prevalence of physical frailty by Fried's criteria in community-dwelling elderly with National Population-Based Surveys. J Am Med Dir Assoc 2015; 16:548-50.

7. Fried LP, Ferrucci L, Darer J, Williamson JD, Anderson G. Untangling the concepts of disability, frailty, and comorbidity: implications for improved targeting and care. J Gerontol A Biol Sci Med Sci 2004; 59:255-63.

8. Vries NM, Staal JB, van Ravensberg CD, Hobbelen JS, Olde Rikkert MG, Nijhuis-van der Sanden MW. Outcome instruments to measure frailty: a systematic review. Ageing Res Rev 2011; 10:104-14.

9. Instituto Brasileiro de Geografia e Estatística. Pesquisa Nacional de Saúde 2013: percepção do estado de saúde, estilo de vida e doenças crônicas - Brasil, Grandes Regiões e Unidades de Federação. Rio de Janeiro: Instituto Brasileiro de Geografia e Estatística; 2014.

10. Mello AC. Aspectos nutricionais, sociodemográficos e de saúde relacionados à síndrome de fragilidade em idosos moradores de Manguinhos, RJ [Tese de Doutorado]. Rio de Janeiro: Escola Nacional de Saúde Pública Sergio Arouca; Fundação Oswaldo Cruz; 2014.

11. Bortz WMA. Conceptual framework of frailty: a review. J Gerontol A Biol Sci Med Sci 2002; 57:M283-8. 
12. Walston J, McBurnie MA, Newman A, Tracy RP, Kop WJ, Hirsch $\mathrm{CH}$, et al. Frailty and activation of the inflammation and coagulation systems with and without clinical morbidities: results from the cardiovascular health Study. Arch Intern Med 2002; 162:2333-41.

13. Fisberg RM, Marchioni DML, Colucci ACA. Avaliação do consumo alimentar e da ingestão de nutrientes na prática clínica. Arq Bras Endocrinol Metab 2009; 53:617-24.

14. Conselho Federal de Nutricionistas. Resolução CFN no 417 de 18 de março de 2008. Dispõe sobre procedimentos nutricionais para atuação dos nutricionistas e dá outras providências. Diário Oficial da União 2008; 24 mar.

15. Mello AC. Aplicabilidade de parâmetros antropométricos e de bioimpedância elétrica na avaliação do estado nutricional de idosos [Dissertação de Mestrado]. Viçosa: Universidade Federal de Viçosa; 2009.

16. Carvalho MS, Mello AC, Rabello, RS. Como está a sua saúde? Resultados da pesquisa sobre a situação de saúde dos moradores da região de Manguinhos. http://www.icict.fiocruz.br/sites/ www.icict.fiocruz.br/files/Saude\%20Urbana_ Projeto\%20Teias_boletim_corrigido.pdf (acessado em 21/Set/2015).

17. Instituto Brasileiro de Geografia e Estatística. Pesquisa Nacional de Saúde. http://www.pns. icict.fiocruz.br/index.php?pag=principal (acessado em 21/Set/2015).

18. Instituto Brasileiro de Geografia e Estatística. Pesquisa Nacional por Amostra de Domicílios. 2009. http://www.ibge.gov.br/estadosat/temas. php?sigla=rj\&tema=pnad_2009 (acessado em 01/Out/2011).

19. Lwanga SK, Lemeshow S. Sample size determination in health studies: a pratical manual. Geneva: World Health Organization; 1991.

20. Radloff LS. The CES-D Scale: a self-report depression scale for research in the general population. Appl Psychol Meas 1977; 1:385-401.

21. Batistone SST, Neri AL, Cupertino APFB. Validade da escala de depressão do Center for Epidemiological Studies entre idosos brasileiros. Rev Saúde Pública 2007; 41:598-605.

22. Organización Mundial de la Salud. El estado físico: uso e interpretación de la antropometría. Genebra: Organización Mundial de la Salud; 1995. (Serie de Informes Técnicos, 54).

23. Gomes MA, Rech CR, Gomes MBA, Santos DL Correlação entre índices antropométricos e distribuição de gordura corporal em mulheres idosas. Rev Bras Cineantropom Desempenho Hum 2006; 8:16-22.

24. Harrison GG, Buskirk ER, Carter JL, Johnston FE, Lohman TG, Pollock ML, et al. Skinfold thicknesses and measurement technique. In: Lohman TJ, Roache AF, Martorell R, editors. Anthropometric standardization reference manual. Champaign: Human Kinetics; 1988. p. 55-70.

25. Gurney JM, Jelliffe DB. Arm anthropometry in nutritional assessment: nomogram for rapid calculation of muscle circumference and cross sectional muscle and fat areas. Am J Clin Nutr 1973; 26:912-5.
26. Ho SY, Lam TH, Janus ED. Waist to stature ratio is more strongly associated with cardiovascular risk factors than other simple anthropometric indices. Ann Epidemiol 2003; 13:683-91.

27. Valdez R. A simple model-based index of abdominal adiposity. J Clin Epidemiol 1991; 44:955-6.

28. Schieri R. Epidemiologia da obesidade. Rio de Janeiro: EDUERJ; 1998.

29. Departamento de Atenção Básica, Secretaria de Atenção à Saúde, Ministério da Saúde. Alimentação saudável para a pessoa idosa: um manual para profissionais de saúde. Brasília: Ministério da Saúde; 2009. (Série A. Normas e Manuais Técnicos).

30. Sousa ACPA, Dias RC, Maciel ACC, Guerra RO. Frailty syndrome and associated factors in community-dwelling elderly in Northeast Brazil. Arch Gerontol Geriatr 2012; 54:e95-101.

31. Vieira RA, Guerra RO, Giacomin KC, Vasconcelos KSS, Andrade ACS, Pereira LSM, et al. Prevalência de fragilidade e fatores associados em idosos comunitários de Belo Horizonte, $\mathrm{Mi}$ nas Gerais, Brasil: dados do estudo FIBRA. Cad Saúde Pública 2013; 29:1631-43.

32. Perez M, Lourenço RA. Rede FIBRA-RJ: fragilidade e risco de hospitalização em idosos da cidade do Rio de Janeiro, Brasil. Cad Saúde Pública 2013; 29:1381-91.

33. Chaves PH, Varadhan R, Lipsitz LA, Stein PK, Windham BG, Tian J, et al. Physiological complexity underlying heart rate dynamics and frailty status in community-dwelling older women. J Am Geriatr Soc 2008; 56:1698-703.

34. Gruenewald TL, Seeman TE, Karlamangla AS, Sarkisian CA. Allostatic load and frailty in older adults. J Am Geriatr Soc 2009; 57:1525-31.

35. Blaum CS, Xue QL, Michelon E, Semba RD, Fried LP. The association between obesity and the frailty syndrome in older women: the Women's Health and Aging Studies. J Am Geriatr Soc 2005; 53:927-34.

36. Shardell M, Hicks GE, Miller RR, Kritchevsky S, Andersen D, Bandinelli S, et al. Association of low vitamin $\mathrm{D}$ levels with the frailty syndrome in men and women. J Gerontol A Biol Sci Med Sci 2009; 64:69-75.

37. Ministério da Saúde. Orientações para coleta e análise de dados antropométricos em serviços de saúde: norma técnica do Sistema de Vigilância Alimentar e Nutricional - SISVAN. Brasília: Ministério da Saúde; 2011.

38. Santos DM, Sichieri R. Índice de massa corporal e indicadores antropométricos de adiposidade em idosos. Rev Saúde Pública 2005; 39:163-8.

39. Jarosz PA, Bellar A. Sarcopenic obesity: an emerging cause of frailty in older adults. Geriatr Nurs 2009; 30:64-70.

40. World Health Organization. Physical status: use and interpretation of anthropometry. Geneva: World Health Organization; 1995.

41. Landi F, Onder G, Russo A, Liperoti R, Tosato M, Martone AM, et al. Calf circumference, frailty and physical performance among older adults living in the community. Clin Nutr 2014; 33:539-44. 
42. Hubbard RE, Lang IA, Llewellyn DJ, Rockwood K. Frailty, body mass index, and abdominal obesity in older people. J Gerontol A Biol Sci Med Sci 2010; 65:377-81.

43. Campos MTFS, Monteiro JBR, Ornelas APRC. Fatores que afetam o consumo alimentar e a nutrição do idoso. Rev Nutr 2000; 13:157-65.

44. Chemim SS, Mura JDP. Tratado de alimentação, nutrição e dietoterapia. São Paulo: Editora Roca; 2007.

45. Departamento de Atenção Básica, Secretaria de Atenção à Saúde, Ministério da Saúde. Guia alimentar para a população brasileira. Brasília: Ministério da Saúde; 2014.

46. Gill TM, Gahbauer EA, Allore HG, Han L. Transitions between frailty states among community-living older persons. Arch Intern Med 2006; 166:418-23.

\section{Abstract}

The aim of this study was to describe anthropometric and food intake data related to the frailty syndrome in the elderly. This was a cross-sectional study in individuals $\geq 60$ years of age in a household survey in the Manguinhos neighborhood of Rio de Janeiro, Brazil $(n=137)$. Frailty syndrome was diagnosed according to Fried et al., anthropometric measures were taken, and a food frequency questionnaire was applied and the results compared to Brazilian Ministry of Health guidelines. In the pre-frail and frail groups, body mass index and measures of central adiposity showed higher levels, while lean muscle parameters showed lower values, proportional to the syndrome's gradation. Frail elderly consumed higher amounts of grains and lower amounts of beans and fruit; pre-frail elderly consumed more vegetables, dairy products, and high-sugar and high-fat foods; the two groups consumed similar amounts of meat. Thus, diagnosis of the syndrome, anthropometric evaluation, and dietary assessment should be included in health policies for the elderly, since they assist in early identification of risk and favor interventions for disease prevention and health and nutritional promotion.

Frail Elderly; Food Consumption; Anthropometry; Nutrition Assessment
47. Espinoza SE, Jung I, Hazuda H. Frailty transitions in the San Antonio Longitudinal Study of Aging. J Am Geriatr Soc 2012; 60:652-60.

48. Lee JSW, Auyeung TW, Leung J, Kwok T, Woo J. Transitions in frailty states among communityliving older adults and their associated factors. J Am Med Dir Assoc 2014; 15:281-6.

49. Starfield B. Atenção primária: equilíbrio entre necessidades de saúde, serviços e tecnologia. Brasília: Organização das Nações Unidas para a Educação a Ciência e a Cultura/Ministério da Saúde; 2002.

50. Departamento de Atenção Básica, Secretaria de Atenção à Saúde, Ministério da Saúde. Política Nacional de Atenção Básica. Brasília: Ministério da Saúde; 2012. (Série E, Legislação e Saúde).

\section{Resumen}

El objetivo de este estudio fue describir datos antropométricos y de alimentación, relacionados con el sindrome de fragilidad en ancianos. El diseño fue transversal, con individuos $\geq 60$ años de una encuesta domiciliaria realizada en Manguinhos, Municipio de Río de Janeiro, Brasil $(n=137)$. Se obtuvo el diagnóstico de síndrome de fragilidad según Fried et al., y las medidas antropométricas, además se administró un cuestionario de frecuencia de consumo alimentario, comparándose con las recomendaciones del Ministerio de Salud. En los grupos pre-frágiles y frágiles, el indice masa corporal y medidas de centralización de grasa presentaron valores más elevados, y los parámetros musculares, valores menores con la gradación del sindrome. El consumo de cereales fue mayor en los frágiles y el de frijoles y frutas menor; el de vegetales, lácteos y alimentos ricos en azúcar y grasa fue mayor en los pre-frágiles; el de carne fue semejante en los grupos. De esta forma, el diagnóstico del síndrome, la evaluación antropométrica y de la alimentación son acciones que deben ser incluidas en las politicas de salud del anciano para identificar precozmente el riesgo y beneficiar intervenciones de prevención y promoción a la salud y nutrición.

Anciano Frágil; Consumo de Alimentos; Antropometría; Evaluación Nutricional
Recebido em 06/Nov/2015

Versão final reapresentada em 04/Set/2016 Aprovado em 22/Set/2016 